\title{
Editorial: Biological Psychiatry/Pharmacopsychiatry
}

The extraordinary development of the neurosciences in recent years has yielded tremendous insight in the understanding of biological correlates of psychiatric disorders and their treatment. This section will publish papers on new methodological advances applied to biological research in behavioral disorders, such as investigations ranging from sleep polygraphic recordings to the study of receptor structure and function, articles on new promising brain imaging techniques such as PET scan and nuclear magnetic resonance, allowing for the first time direct and noninvasive exploration of the brain. Change is rapid in this new field, making it almost impossible to acquire an expertise in the various areas of psy-chobiological research. There is also good evidence from animal and clinical studies that genetic biological factors may interact with environmental factors both at the molecular and supramolecular level. This interrelationship between nature and nurture is of particular interest today for brain research, and our Biologica Psychiatry section will promote such multidisciplinary studies among its goals. This section will provide rapid publication for original research reports relevant to biological psychiatry: biochemistry, physiology, genetics, endocrinology, epidemiology, pharmacology, and related disciplines. New reports of basic research studies of clinical interest will also be published as well as new advances in methodology in relation to biological psychiatry. Book reviews and review articles of particular interest to the field will also be considered. 
The extraordinary development of pharmacopsychiatry in recent years has opened new ways in the understanding and treatment of disturbed human behaviour. In addition to their importance in the treatment of psychiatric patients, psychotropic drugs have vigorously catalysed research into the biological determinants of mental disorders.

Major advances have been made in the methodology of psychotropic drug research. Sophisticated experimental designs and guidelines have been developed. New instruments have been presented for the evaluation of signs and symptoms. Operational diagnostic criteria for inclusion and exclusion have been elaborated. Particular attention is being paid to ethical issues.

The Pharmacopsychiatry section intends to keep the reader in touch with psychotropic drug research and its potential practical applications. The journal will provide rapid publication for a wide range of reports concerning basic research as well as clinical investigations in pharmacopsychiatry. In addition to reports on new developments in the field, the section will feature state-of-the-art reviews by experts. Short communications on recent advances in basic and clinical psychotropic drug research will be considered.

Particular attention will be given to the methodology of clinical investigations on human subjects. Reports submitted for publication in the Pharmacopsychiatry section should be based on modern designs. Precise references should be made to reliable criteria for inclusion and exclusion of subjects. Evaluation of change should be based on instruments that have been validated. Reports should systematically include clear statements concerning informed consent by the patients. 